\title{
Linx
}

Revue des linguistes de l'université Paris X Nanterre

$82 \mid 2021$

Entre vieillissement et innovation : le changement linguistique

\section{Asteure, archaïsme et changement linguistique : éclairages réciproques des français nord- américains et des français de France}

Asteure, archaism and linguistic change: mutual perspectives in American

French and French from France

\section{Cristina Petraș}

\section{OpenEdition}

Journals

Édition électronique

URL : https://journals.openedition.org/linx/8025

DOI : 10.4000/linx.8025

ISSN : 2118-9692

Éditeur

Presses universitaires de Paris Nanterre

Référence électronique

Cristina Petraș, «Asteure, archaïsme et changement linguistique : éclairages réciproques des français nord-américains et des français de France », Linx [En ligne], 82 | 2021, mis en ligne le 15 juillet 2021, consulté le 21 juillet 2021. URL : http://journals.openedition.org/linx/8025 ; DOI : https://doi.org/ 10.4000/linx.8025

Ce document a été généré automatiquement le 21 juillet 2021.

Département de Sciences du langage, Université Paris Ouest 


\title{
Asteure, archaïsme et changement linguistique : éclairages réciproques des français nord- américains et des français de France
}

\author{
Asteure, archaism and linguistic change: mutual perspectives in American \\ French and French from France
}

Cristina Petraș

\section{Introduction ${ }^{1}$}

1 Dans le contexte d'une approche du vieillissement des faits de langues, de l'innovation et du changement linguistique, envisager les rapports entre français de France et français hors de France s'avère particulièrement riche. En effet, les faits de langue transportés ailleurs subissent souvent des évolutions, des reconfigurations, des dynamiques, dont l'analyse peut apporter des éléments à une théorie du changement linguistique. Il en est ainsi de la forme asteure, qui constitue l'objet de cet article. D'emblée il faut préciser que les déplacements formels et sémantiques d'asteure observés dans les français nord-américains ne constituent pas un cas de figure isolé. D'autres marqueurs (ça fait que/fait que/fak, pis, là) se comportent de la même manière. Ils font d'ailleurs l'objet d'une littérature abondante, pour ne citer que Dostie (2004b, 2007, 2015), Falkert (2006) et Petraș (2016).

2 Largement attesté et bien documenté de nos jours dans les variétés de français nordaméricain, comme en témoignent les corpus et les dictionnaires, l'adverbe asteure ${ }^{2}$ est un héritage de France, où il est devenu vieilli, après avoir connu un emploi considérable à partir du moyen français tant chez les différents auteurs que dans les parlers d'oïl (voir FEW, $4: 468$; DMF). Selon (Arrighi, 2005), il se maintient en français régional de France et en Belgique francophone. 
3 En comparant les emplois enregistrés dans les français nord-américains - en synchronie et en diachronie - à ceux des français de France, on remarque le fonctionnement d'asteure comme marqueur discursif dans les français nord-américains contemporains, emploi plus rare dans les français de France.

4 L'équivalent du français de référence proposé le plus souvent pour rendre compte du sens de asteure est maintenant ${ }^{3}$, qui est rare, voire absent dans certains des corpus de français nord-américains consultés. Une analyse comparée de désormais, dorénavant et asteure, qui prend forcément en compte la dimension aspectuo-temporelle, conclut à l'existence de convergences comme de divergences du point de vue de leurs fonctionnements respectifs (Séguin, 2011).

5 À la lumière des constats ci-dessus, nous nous interrogerons sur les mécanismes et le parcours du changement sémantique que connaît asteure dans les variétés de français nord-américain par rapport à son emploi en France (et implicitement sur le rapport entre asteure et à cette heure). Analyser les combinaisons d'asteure avec différents marqueurs (par exemple, là asteure, asteure là) permet une approche du figement dans les associations de marqueurs, question qui est consubstantielle à l'émergence de certains marqueurs et au mécanisme de pragmaticalisation (voir à cet égard Dostie, 2004a).

6 Notre démarche s'appuiera, d'une part, sur des corpus oraux de français nordaméricains, ainsi que sur des corpus de contes de tradition orale acadiens, et, d'autre part, sur des corpus de textes de français de France de différentes époques.

7 La première section sera consacrée à la description des emplois d'asteure et de ses associations dans les français nord-américains contemporains. Étudier la manière dont les dictionnaires anciens recensent les emplois d'asteure permet d'envisager une approche historique. La deuxième partie présente la dynamique et l'évolution des expressions à cette heure et asteure dans les français de France, telles qu'elles se dégagent à partir d'une analyse qui exploite les données offertes par Frantext, par les glossaires régionaux et par la littérature régionale. La comparaison des deux situations (français nord-américains et français de France) permet de tirer des conclusions concernant l'émergence d'asteure et son évolution vers l'emploi comme marqueur discursif.

\section{Ast(h)eure dans les français nord-américains}

8 Les analyses proposées exploitent notamment des corpus oraux des années 2000. Quelques-uns des enregistrements du corpus Arrighi (2005) datent des années 1980 et 1990. Ce sont des enregistrements faits dans un but ethnographique. Pour ce qui est du corpus de contes de tradition orale que nous avons également exploité, celui-ci couvre la seconde moitié du XX $\mathrm{XX}^{\mathrm{e}}$ siècle. Le Fichier lexical du Trésor de la Langue Française au Québec (TLFQ) s'appuie sur des documents datant d'époques différentes, s'étendant du $\mathrm{XVI}^{\mathrm{e}}$ au $\mathrm{XXI}^{\mathrm{e}}$ siècle. Les corpus oraux ont été constitués par la collecte de données sollicitées - entretiens semi-directifs (Arrighi, 2005 ; Falkert, 2010 ; Wiesmath, 2006) ou de données écologiques - interactions verbales dans des contextes informels (Corpus de français parlé au Québec) (CFPQ), émissions de radio communautaire (Petraș, 2016). TLFQ couvre des genres textuels différents (littérature, textes humoristiques, satiriques 
dans la presse, documents ethnographiques, enquêtes linguistiques, littérature personnelle, documents d'archives, textes spécialisés, etc.).

\subsection{Significations et emplois}

9 Les emplois d'asteure dans les variétés de français nord-américain peuvent être rapprochés des emplois de maintenant. En suivant l'approche que Bertin (2001) propose des valeurs de maintenant en diachronie, nous identifions une "valeur temporelle » et un emploi comme « adverbe de relation » ou marqueur discursif.

\subsubsection{Asteure temporel}

10 a) On pourra identifier un premier asteure [asteure ${ }_{1}$ ] adverbe de constituant, qui comporte une signification déictique, pouvant être paraphrasée par « maintenant, à cette heure, en ce moment", comme dans les exemples (1), (2), (3) et (4). La construction conjonctive asteure que est aussi attestée (5).

(1) $\mathrm{H}^{9} 1$ : [...] et h'avais demandé à $\left[\mathrm{P}_{1}\right]$ de revenir c'te semaine / pour nous donner manière d'un compte rendu point en détail / mais rinque nous donner un petit compte rendu de comment-ce que c'te session là avait été et $\left[\mathrm{P}_{1} \mathrm{~N}_{1}\right]$ est dans le studio asteure / $\left[\mathrm{P}_{1}\right]$ comment ça va (corpus Petraș, 2016)

(2) $\mathrm{H}^{5} 2$ : ça fait qu'y a des endroits que la CENTRAL LINE / c'est encore / on oit la CENTRAL LINE mais qu'y a encore de la glace dessus

$\mathrm{H}^{5} 1$ : ouais ouais

$\mathrm{H}^{5} 2$ : euh plus' plus' du bout' de Meteghan en allant par Comeauville et ça il est tout' pas mal CLEAR / autre que pour la poudre qu'est en frais de / couvrir BACK dessus asteure

$\mathrm{H}^{5} 1: \mathrm{hm}$

$\mathrm{H}^{5} 2$ : c'est point / là / c'tes endroits-là / c'est point / c'est point trop coulant (corpus Petraș, 2016)

(3) $\mathrm{H}^{10} 1$ : le saxophone / et / et le piano aussi euh quoi-ce que / quand-ce que tu gardes l'album toi asteure / quoi-ce que tu dirais qu'est / est / est / qui va être ton souvenir de c't album / quoi-ce qui te sort le plus toi (corpus Petraș, 2016)

(4) - M : mais c'est pas ${ }^{1}$ nécessairement une mauvaise ${ }^{2}$ chose euh: dans un sens c'est pas nécessairement mauvais aus- non plus d'avoir une opinion sur TOUT là/ c'est c'est juste c'est l- de la manière qu'on s'exprime de la manière \{que euh;que:\} qu'on la partage notre opinion astheure qui est qui est rendu le principal problème ${ }^{3}$ moi je trouve [sous-corpus 30, segment 9, page 117, ligne 13] (CFPQ)

(5) $\mathrm{H}^{26} 1$ : SO pour deux mille quatre bien si que / si tu regardes ça là asteure que / la poussière est settlée là (corpus Petraș, 2016)

11 Si asteure exprime un rapport de simultanéité par rapport à la référence qui est le moment de l'énonciation, la nature de ce rapport est assez ambiguë. La simultanéité s'établit parfois avec un moment du dire flou, de durée plus ou moins étendue (voir les exemples $6,7,8,9)$. C'est ce qui justifie un élargissement du sens déictique, asteure signifiant aussi " de nos jours, ces temps-ci », selon Neumann-Holzschuh et Mitko (2018: 779).

(6) $\mathrm{F}^{2} 1$ : ça fait que es-tu au courant de you-ce que je serions rendus / pour notre province / quoi-ce qui est en train de se passer là-dedans asteure ou $\mathrm{F}^{2} 2$ : pour la région ici là / comme ça que je / je te dis ça je sais là $\mathrm{F}^{2} 1$ : ouais 
$\mathrm{F}^{2} 2$ : tu sais je sais qu'y a des comités y a du monde /qui, qu'i/ travaille là-dedans / et / et ça / et pis la province je crois qu'i sont vraiment contents des des quatorze / projets / là qu'il ont avec des / des NURSE PRACTITIONER (corpus Petraș, 2016)

(7) $\mathrm{F}^{4} 2$ : USUALLY c'est entre / cinq à quarante / comme alentour de trente ans / mais dernièrement i trouvont plus' en plus' des jeunes / qui sont diagnosés $\mathrm{F}^{4} 1$ : oh y a plus' de jeunes asteure

$\mathrm{F}^{4} 2$ : plus' asteure ouais que dernièrement (corpus Petraș, 2016)

(8) $\mathrm{F}^{13} 2$ : $\mathrm{y}$ a des mères et des pères qui venont asteure qu'avont venu à [activité] pis venont avec leur enfant (corpus Petraș, 2016)

(9) $\mathrm{H}^{25} 1$ : mais quand j'étions petits BOYS et ça ça me ressemble h'avions des plus' gros hivers que h'avons asteure (corpus Petraș, 2016)

L'interprétation proposée montre l'équivalence de fonctionnement entre asteure et maintenant, le dernier ayant été étudié par Bertin (2001) déjà citée. Selon cette même auteure, en ancien français, c'est or qui assure la fonction déictique, alors que maintenant fonctionne comme adverbe de constituant, exprimant l'idée d'immédiateté. Maintenant se substituera à or comme déictique en moyen français.

b) Deuxièmement, on constatera dans certains contextes un glissement sémantique lié au changement de référence (déictique/anaphorique) correspondant à la signification anaphorique (" alors, à cette époque-là, en ce temps-là ", voir Neumann-Holzschuh et Mitko, 2018 : 779) comme dans les exemples (10), (11), (12) et (13) ([asteure $\left.\left.{ }_{2}\right]\right)$. Le même emploi est enregistré par Bertin (2001) pour maintenant dès le moyen français.

(10) $i$ aviont pas des grosses pensions asteure peut-être ben vingt piasses ou trente piasses (corpus Arrighi, 2005)

(11) $\mathrm{F}^{13} 2$ : et / euh comme ç'a changé depuis que c'était / comme quand j'ai commencé [activité] / y avait beaucoup de: / de / de mères qui venaient / qui travailliont point asteure / eh ben on oit que n'y a

$\mathrm{H}^{13} 1$ : ça a changé

$\mathrm{F}^{13} 2$ : un grous grous change / on oit le change / on oit un grous change (corpus Petraș, 2016)

(12) 0 : il le voyait pis le soir il est arrivé les cennes noires il en avaient plus une crisse à la même place (en passant son index sur la table comme pour montrer le déplacement des cennes) $\langle\mathrm{P} 28, \mathrm{~L} 16\rangle$

$\mathrm{Y}:$ ah $\left[{ }^{1}<\mathrm{p}<\{\right.$ fait qu'il y en avait qui;(inaud.) $\}>><\mathrm{P} 28, \mathrm{~L} 17>$

$0:\left[{ }^{1}\right.$ pis astheure il partait travailler avec tout son argent tous ses papiers (en pointant son pouce vers l'arrière comme pour désigner l'endroit où son frère partait) $<$ P28,L18 $>$ (CFPQ)

(13) Le vieux approchait de ses soixante-dix astheure pis i commançait à courber comme un âbre avec des racines qui poignent pus dans la vieille terre du pays. Le vieux s'en alllait. (TLFQ, 1945, Claude-Henri Grignon, Le père bougonneux, in Bulletin des agriculteurs, Montréal)

14 Asteure s'associe dans ces cas avec l'imparfait dans sa dimension anaphorique, telle qu'envisagée, parmi d'autres, par Berthonneau et Kleiber (1993), Kleiber (2003).

\subsubsection{Asteure marqueur discursif}

15 Comme maintenant, asteure fonctionne dans les français nord-américains contemporains comme marqueur discursif [asteure ${ }_{3}$ ]. Cet emploi est différemment paraphrasé dans les travaux qui le recensent: «donc, au point où l'on est/était » (Neumann-Holzschuh et Mitko, $2018:$ 779) et «aussi, en plus» (Cormier, $1999: 71)$. En outre, Brasseur (2001: 31) utilise pour en rendre compte justement la paraphrase donnée par TLFi pour maintenant: « mot qui "suggère la pertinence de l'énoncé qui le 
comporte au point du discours où on est parvenu" (TLF 11, 190a: maintenant)». Dans son emploi comme marqueur discursif, asteure correspond en outre à « ceci (étant) dit ». Ce type d'emploi n'est pas enregistré dans les inventaires lexicographiques de français nord-américains plus anciens.

On peut envisager plus largement une grammaticalisation qui suppose un déplacement d'un sens temporel vers le sens anaphorique, de relation, qui caractérise plusieurs adverbes de temps ${ }^{4}$. Ainsi, asteure présente un comportement proche de maintenant (comme déjà mentionné ci-dessus) et or, analysés par Bertin (2001). Il s'agit d'indiquer que l'énoncé introduit renvoie à ce qui précède, le marqueur prenant une valeur adversative. Tout le mouvement de grammaticalisation de maintenant décrit par l'auteure citée a, à la base, des relations de type métonymique ${ }^{5}$.

Nous avons identifié plusieurs types d'emplois d'asteure comme marqueur discursif. Ainsi, il peut intervenir pour marquer le changement de thème. Dans le corpus Petraș (2016), ce type d'emploi est notamment l'apanage des animateurs, du fait de leur rôle dans l'échange et dans l'organisation du discours (voir 14).

(14) $\mathrm{H}^{12} 1$ : So quéqu'un qui prendra la peine d'acheter c'tes huit heures icitte / pis qu'est sus le STUFF: [toussote] qu'est sus leu chanson / leu-z-instrumental WHATEVER [toussote] / ça / i pourriont presque / le faire dans deux je crois ben $\mathrm{H}^{12} 2$ : ça viendra moyennement proche

$\mathrm{H}^{12} 1$ : ça viendra $\mathrm{X}$

$\mathrm{H}^{12} 2$ : ouais quéqu'un qui peut jouer ses chansons ieune après l'autre $\mathrm{XX} /$ et pis que n'y a point de problème là / ouais / ouais ça pourrait être ben intéressant

$\mathrm{H}^{12} 1$ : asteure quoi-ce qu'arrive si que tu commences un projet / et / pour un album je dirais pis tu veux faire une douzaine de chansons / et pis que tu dépasses tes huit heures / euh coum- coumment-ce qu'on s'arrange après / y a-ti des arrangements après ça ou / i payereriont peut-être SO MUCH LOW pour finir le projet ou (corpus Petraș, 2016)

Avec cette valeur asteure occupe généralement la position en tête d'énoncé. Pourtant, dans (15), par exemple, il est placé à la fin de l'énoncé. D'ailleurs, le marqueur so, qui figure en tête d'énoncé, intervient lui aussi comme marqueur de changement de thème.

(15) $\mathrm{F}^{7} 2$ : et pis / ç'a vraiment ben été / h'avons vu que que / les femmes workiont ben ensemble / ben i nous avont dounné des vraiment bonnes suggestions / pis h'avons eu les FEEDBACK que h'avons eu nous autres c'est que ouais / les femmes / entrepreneurs / pis les femmes professionnelles de la région de Clare voulont un groupe comme cecitte

$\mathrm{H}^{7} 1: \mathrm{hm}$

$\mathrm{F}^{7} 2$ : ça fait / nous autres h'allons aller de l'avant avec zeux / pour / pour en organiser en organiser ène : ène série et pis assayer de / de coummencer ça

$\mathrm{H}^{7} 1$ : WOW / pis ç'a duré une couple d'heures ou

$\mathrm{F}^{7} 2$ : ouais / h'avons sorti le temps qu'était il était une miette passé neuf heures là /

so

$\mathrm{H}^{7} 1$ : SO quoi-ce qu'est la prochaine étape asteure

$\mathrm{F}^{7} 2$ : WELL / la prochaine étape c'est h'allons prendre les recommandations /

h'allons prendre les suggestions que les femmes avont fait (corpus Petraș, 2016)

On pourrait aussi comparer cet emploi d'asteure à celui plus large comme déictique textuel de là et comme marqueur discursif, plus particulièrement (Dostie, 2007), que Wissner (2017) appelle " marqueur discursif de suite ». Le rôle des marqueurs comme là, puis, pis, fait que, alors dans l'organisation du discours permet à Dostie (2007) de parler d'un emploi méta-discursif. 
20 L'emploi comme marqueur discursif d'asteure est compatible avec le fonctionnement du récit. Dans (16), tiré d'un conte acadien de tradition orale, la première occurrence d'astheur introduit une séquence permettant la compréhension de la séquence antérieure, tout en indiquant la pertinence de la séquence introduite dans l'économie du récit. Astheur pourrait être paraphrasé dans ce cas de la manière suivante : « arrivé à ce moment de l'histoire, il faut dire que...». La deuxième occurrence intervient dans une séquence qui fournit le cadre, l'arrière-plan, introduisant un élément nouveau, qui facilitera à son tour la compréhension de la séquence narrative suivante.

(16) Ça fait ti-Jack lui s'en a 'té, pis il a 'té su sa tante, pis i a trouvé une pelle pis i l'a r'tourné back au champs, pis quand qu ['] i a arrivé au champs, les sept têtes étiont parties. I avait pus 'ien qu'le corps ï a trouvé ça drôle, astheur c'tait 1'baron ça qu'avait envoyé les valets du roi chercher les têtes pour les rapporter à la cave du vieux roi pour la journée des noces pour montrer ça au monde.

Ça fait o.k.

Ben la fille ielle (s'avère la langue?) qu'c'tait point lui. A savait qu'c'était p'tit Jack qui l'avait sauvée.

Ben toujou' p'tit Jack lui, a'té là pis il a enterré la bête, il a arrangé ça comme il faut pis i a r'tourné back su' sa tante.

Ça fait, ça va vite dans les contes, une bonne journée, c'était les noces. Astheur, la coutume par là, i faisiont leur r'pas avant d'se marrier.

Ouais sa tante était pas invitée, lui non plus, pis toutes l'autre...les autres mondes étaient invités aux noces ${ }^{6}$.

21 Avec l'exemple (17) astheur comporte une valeur légèrement différente. Les deux occurrences apparaissent en alternance avec là (le premier) pour assurer la cohérence du récit.

(17) Ça fait i l'a rentré son joual en'dans pis z-eux s[']avont halé d'son côté, là i a rentré à la maison, astheur, i s'greyont pour s'mettre à table, à la table, astheur il a vu là ... le... le l'chose qu'était là, le... ah! comment j'vas...?

\subsection{Asteure dans les corpus nord-américains et dans les dictionnaires}

Dans certains des corpus de français acadien consultés, ast $(h)$ eure et la construction asteure là ${ }^{7}$ sont présents à côté de maintenant et de la construction maintenant là (Arrighi, 2005, Falkert, 2010). Dans le corpus de Wiesmath (2006), il se manifeste une répartition assez claire entre maintenant et asteure, les locuteurs utilisant l'un ou l'autre (mais surtout asteure) en fonction de facteurs sociaux (niveau socio-économique, métier - un photographe ou des responsables administratifs utilisent maintenant). Dans le corpus Petraș (2016), une seule des locuteurs/locutrices employant maintenant utilise aussi asteure. Trois autres n'utilisent que maintenant. Hormis les locuteurs cités (invités dans les émissions) et les deux animateurs (chez qui on remarque un emploi considérable de là asteure), la quasi-totalité des autres locuteurs utilisent asteure dans tous les emplois. Dans le même corpus, des adverbes comme désormais et dorénavant sont totalement absents.

Asteure est également bien attesté en français québécois dans tous les emplois, comme il ressort du Corpus de français parlé au Québec (CFPQ) et du Fichier lexical du Trésor de la Langue Française au Québec (TLFQ). Maintenant est aussi présent. Dans l'exemple (18), les deux apparaissent dans des emplois différents : astheure temporel, maintenant marqueur discursif. 
(18) L : <len<ils sont venus au monde aux États-Unis (.) là ils avaient DEUX ans (.) pour demander leur citoyenneté canadienne (1,3") pour avoir la la c:itoyenneté canadienne pis américaine (.) mais le monde ils savent pas ça (.) là ils arrivent à cinquante ans astheure maintenant pour aller aux États-Unis ça te prend $>>($. $)<\mathrm{f}<\mathrm{un}$ passeport $>>(0: 02: 01.0)<\mathrm{P} 93, \mathrm{~L} 10>(\mathrm{CFPQ})$

a profondeur diachronique de la masse de documents couverts par le Fichier lexica du Trésor de la Langue Française au Québec, une brève analyse des différentes formes attestées (asteur, asteure, astheur, astheure, à c't'heure, à cette heure) permet d'avancer des hypothèses concernant l'évolution et l'émergence de l'adverbe. Ce qu'on remarque, c'est une coexistence de ces différentes formes au $\mathrm{XX}^{\mathrm{e}}$ siècle, alors que certaines semblent plus anciennes que d'autres. Ainsi, à cette heure, avec le sens grammaticalisé " maintenant », est enregistré déjà dans les récits de Marie de l'Incarnation, en 1639 et 1647 (voir 19 ci-dessous). La forme se maintient jusque dans les années 2000.

(19) Nous avons tous ressenti de mal de la mer ; mais cela n'est rien : Nous sommes à cette heure dans une aussi bonne disposition que si nous étions dans notre Monastère (TLFQ, 1971, Marie de l' Incarnation Ursuline (1599-1672): correspondance, nouv. éd. par Dom Guy Oury, Solesmes, Abbaye Saint-Pierre, LXVI-1075., p. 86 ; correspondance datant de 1639)

La forme à c't'heure est attestée avant asteur, asteure, astheur et astheure, soit à partir de 1808, les autres étant enregistrées à partir respectivement de 1843, 1918, 1939 et 1914. Ceci semble indiquer la reconnaissance, par l'intermédiaire de l'orthographe, de l'émergence d'une forme nouvelle, d'un sens unitaire, différente de la construction à cette heure.

Pour ce qui est de la présence dans les dictionnaires, asteure est pris en compte tant par les dictionnaires de la seconde moitié $\mathrm{du} \mathrm{XIX}^{\mathrm{e}}$ siècle et de la première moitié du $\mathrm{XX}^{\mathrm{e}}$ siècle, que par ceux autour des années 2000 . Toutes les significations ne sont pourtant pas recensées dans tous. Si asteure est attesté dans les dictionnaires anciens (Dunn, 1880 ; Clapin, 1894 ; Dionne, 1909 ; Glossaire du parler français au Canada, 1930 ; Poirier (1993 : 36b) [1885]), la signification discursive ne semble pas être prise en compte, alors qu'elle est bien présente dans la première moitié $\mathrm{du} \mathrm{XX}^{\mathrm{e}}$ siècle, comme on le remarque, par exemple, dans (20) et (21) prélevés dans le Fichier lexical. Ces exemples datent respectivement de 1919 et de 1931.

(20) Wilson, [...], c'est pas un poisson ordinaire. [...] Vous allez voir, s'il va vous twister l'Angleterre. Asteure, il n'est peut-être pas ben chum avec Clémenceau. Attendez, il va vous sortir une patente qui arrangera cette affaire-là (TLFQ, Nézyme, 4 janvier, p. 11, 1919, texte humoristique, satirique)

(21) Tu ne t'occupes que de toi, et tu peux double-crosser tout le monde. A c't'heure,

[...] donne-moi d'l'argent (TLFQ, 1931, Le Goglu, texte humoristique, satirique)

Même certaines des attestations de la construction à cette heure semblent indiquer une signification discursive dans le Fichier lexical.

L'adverbe asteure est présent aussi dans les dictionnaires plus récents. Ainsi, Brasseur (2001 : 31) l'enregistre (y compris avec un sens discursif) pour Terre-Neuve (avec steure aussi). Le «type lexical » est également cité pour le Canada, la Louisiane et les créoles à base française. Cormier (1999: 70b-71a), à son tour, recense tant la valeur déictique, que le sens discursif. 


\subsection{Asteure et ses associations}

apparait souvent, dans les corpus soumis à l'analyse, en at d'autres éléments (là, droit, fait que, t'sais). Nous analyserons le degré de figement de ces combinaisons en suivant Dostie (2013) et Waltereit (2007). La question de la combinaison des marqueurs a été examinée par Dostie (2013) dans le cadre plus large de la phraséologie. Ainsi identifie-t-elle, en fonction du degré de figement et plus particulièrement de l'autonomie sémantique des unités composantes, trois types d'associations des marqueurs discursifs, du moins figé vers le plus figé : cooccurrences discursives libres (associations libres, caractérisées par l'autonomie sémantique des éléments); collocations discursives (semi-figées, l'une des unités - sémantiquement autonome - agit comme marqueur-tête, venant sélectionner l'autre marqueur); locutions discursives (figées ; les éléments juxtaposés correspondent à une entité en soi au plan sémantique). Waltereit (2007) envisage les combinaisons de marqueurs comme des "marqueurs discursifs complexes", en distinguant entre les combinaisons libres dans le discours et les combinaisons stockées dans le lexique.

Asteure s'associe dans les corpus consultés fréquemment avec là dans deux configurations - asteure là et là asteure. Pour ce qui est de la signification, on remarquera que la première fonctionne comme déictique (voir les exemples de 22 à 24 ).

(22) $\mathrm{H}^{15} 2$ : je sais point juste combien-ce que n'y en a / parce que je suis en frais de collecter asteure là // et h'allons aoir / h'avons un demi-cochon et pis une couverte QUEEN SIZE (corpus Petraș, 2016)

(23) $\mathrm{M}$ : ${ }^{1}$ pis là j'ai si- j'ai signé full papiers là c'est vraiment épeurant là avant ils faisaient pas ça là avant c'était comme plus euh je sais pas là ils étaient ${ }^{2}<$ rall<plus relax avec ça là > $\mathrm{t}-<\mathrm{p}<\mathrm{je}$ sais pas là>> c'était comme normal mais astheure là ils se prot- à cause qu'il y a des poursuites pour tout là $<p<$ de nos jours anyway >> fait que fallait qu'on: que je signe un papier pis là [sous-corpus 10, segment 2, page 20, ligne 12] (CFPQ)

(24) comme asteure là je sais pas si a' me comprend ielle (corpus Wiesmath, 2006). Holzschuh et Mitko (2018: 779) considèrent que là vient renforcer la valeur déictique d'asteure. Ceci est possible grâce à la valeur de déictique temporel que revêt là lui-même (Dostie, 2007). En effet, ce qui semble favoriser l'association en question, c'est la compatibilité sémantique des deux éléments. D'autre part, Wissner (2017: 89) soutient que là fonctionne dans ce cas comme « marqueur discursif de clôture » qui « clôture un adverbe de lieu ou de temps ", dont asteur).

L'association là asteure semble plus rare que asteure là. Elle fonctionne à la fois comme déictique et comme marqueur discursif. Nous l'avons repérée dans le corpus Petraș (2016) et dans le corpus de contes de tradition orale acadiens. Dans le premier, elle est employée par les animateurs d'émissions comme marqueur d'ouverture qui vient introduire la contribution du locuteur à l'échange (voir 25 et 26).

(25) $\mathrm{F}^{3} 4$ : c'est de même que je nous avons aperçu / que MUM itou faisait c'tes gestes-là que j'avons realizé que c'était ça qu'a faisait / quand-ce qu'alle a dit j'ai dit ça c'est ta fille $\left[\mathrm{P}_{7}\right]$ MUM qui reste là pis a savait point a s'en souvenait pus l'affaire du passé

$\mathrm{F}^{3} 1$ : là asteure a s'en arait point souvenu / alle a-ti réalisé que n'y avait eu / ène manque là / alle l-a-ti réalisé qu'a s'en avait pus souvenu (corpus Petraș, 2016)

(26) $\mathrm{F}^{9} 2$ : ça c'est une discussion pour un autre jour parce que là / h'arais des informations que je pourrais partager avec toi itou $\left[\mathrm{P}_{2}\right]$ 
$\mathrm{H}^{9} 1$ : là asteure pour / pour ça qu'a été discuté / samedi / je m'imagine qu'y arait un rapport / officiel /qui, qu'i/ va sortir (corpus Petraș, 2016) un échange le discours d'un personnage (en report direct).

(27) - Oui, ah ! bien, on va t'engager. On va l'dire au roi pis à la reine. I allont au roi pis à la reine pis i contont ça.

- Ah! ben engagez-les!

I 1'avont engagé.

- Là i avont dit là astheur tu iras à une telle porte, tel nombre de porte. Pis là, tu iras 'oir quoi c'qu'a veut (coll. Florida Arseneault Landry, inf. Virginie Bastarache, 1971, Saint-Thomas, Kent, bob. 1-2, enreg. 3).

Il s'agit là de l'emploi méta-discursif qui a déjà été évoqué (voir 1.1.2.) (paraphrasable par « arrivé à ce moment du discours, je viens/peux affirmer, demander »). Pour ce qui est de la manière dont se construit le sens de cette association, on remarque une accumulation des sens des deux éléments composants, qui sont assez proches.

Quant au caractère libre ou lié des deux associations analysées, si l'on suivait la typologie proposée par Dostie (2013), on pourrait les considérer comme des locutions discursives: les significations des deux marqueurs qui composent ces associations convergent vers un sens unitaire. Dans un autre type d'approche, Waltereit (2007) déjà cité se sert de la lexicalisation (dans le sens d'une inscription dans le stock lexical) pour différencier entre une combinaison liée (stockée dans le lexique) et une combinaison libre. Les constructions ast(h)eure là et là ast(h)eure peuvent être considérées comme faisant partie du stock lexical, puisqu'elles sont reproduites par les locuteurs: il ne s'agit pas d'associations isolées, singulières, qui ne se reproduiraient pas dans d'autres situations de discours ou chez d'autres locuteurs.

La construction là asteure ne fonctionne pas dans tous les contextes comme marqueur discursif. Dans les exemples (28) et (29), là asteure apparaît plutôt comme une variante de asteure là analysée ci-dessus, asteure présentant une valeur déictique renforcée par là.

(28) pis là asteure ça existe plus (corpus Wiesmath, 2006)

(29) SO : ${ }^{2}$ non non non c'est t'sais il pousse pas il serait capable il a le talent là mais il pousse pas fait que là c'est ça l'a- l'art plastique nous-autres on dit tout le temps là $\cdot$ il est beau ton dessin ah c'est beau continue gna gna ${ }^{\circ}$ là astheure on fait plus ça c'est plate là mais il fait un dessin j'ai dit •ben là je m'excuse ça a l'air d'un dessin de maternelle ${ }^{\circ}$ [sous-corpus 7, segment 9, page 100, ligne 11] (CFPQ)

La coexistence de deux occurrences de là dans un même contexte immédiat en (30) tant antéposé que postposé à astheure - pourrait résulter d'un effort d'étoffement, propre à la dynamique du discours oral.

(30) $\mathrm{C}$ : ouin ben c'est ça c'est-tu <f<pire>> 1 ou si c'était comme avant/ c'est c'est ${ }^{2} \mathrm{SU}: \mathrm{R}$ que là astheure là avec la communication qu'il y a c'est telleMENT euh: a<131435> [sous-corpus 1, segment 2, page 15, ligne 17] (CFPQ)

Outre les deux associations déjà analysées, on en enregistre une troisième dans le corpus Petraș (2016) de français acadien de la Nouvelle-Ecosse. L'association droit asteure fonctionnant comme déictique est proche de l'anglais right now, ce qui pourrait indiquer un calque (voir 31).

(31) $\mathrm{F}^{16} 2$ : c'est ça fait point de différence si que c'est quelqu'un qui / vient icitte en train de visiter droit asteure qui qui demeure à Halifax ou de quoi (corpus Petraș, 2016) 
D'ailleurs, right est enregistré par Chevalier et Hudson (2005: 292) comme intensificateur en chiac dans right asteur, right là, right icitte ${ }^{8}$. Ces constructions sont mises en rapport avec right now, right there, right here. Les mêmes auteures considèrent right comme un emprunt qui tend à s'autonomiser en chiac, ce dont rendent compte ses emplois multiples. Une transposition partielle semble avoir eu lieu dans la construction droit back, enregistrée dans le corpus Arrighi (2005) (voir 32).

(32) quand il a té en haut il a dit ielle s'en a retourné droiT BACK en bas (corpus Arrighi, 2005)

Dans le corpus Petraș (2016) la construction droit asteure est introduite aussi par comme (voir 33 et 34), qui connaît en français acadien un emploi de marqueur discursif.

(33) $F^{2} 1$ : d'abord si qu'on se mettait ensemble icitte pis qu'on fait une demande / pis si qu'on voudrait avoir une clinique / une clinique avec toutes sortes de monde(s) dedans / comme droit' asteure au centre médical n'y a beaucoup de monde (corpus Petraș, 2016)

(34) $\mathrm{F}^{16} 2$ : c'est / i regardont quand-ce que / i regardont sus les / les INCOME TAX RETURN de / coumme droit asteure ça sera deux mille trois point deux mille quatre / deux mille trois (corpus Petraș, 2016)

41 Ast(h)eure figure aussi dans des associations libres : il en est ainsi de fait que t'sais astheure ou fait que là astheure t'sais là dans (35), (là) là astheure ou t'sais astheure dans (36), ben vraiment asteure là dans (37), pis là astheur dans (38), ben là astheur, dans (39). Les marqueurs qui s'associent librement dans le discours apportent leur propre signification à l'interprétation.

(35) I : ${ }^{4}$ non mais c'est ça mais là t'a:s t'as ${ }^{5}$ t'as à gérer les: les deux dans cet ${ }^{6}$ apprentissage-là tandis que t'sais MOI j'ai eu de la chance d- d'entraîner Alfred là-deDANS fait que t'sais astheure à pied ou en vélo ça s'appelle 7.tu t'en vas sur le côté fait que là astheure t'sais là ${ }^{8}$ je suis en apprentissage avec Françoi:se ${ }^{9}$ là $<\mathrm{p}<\mathrm{t}$ 'sais >> [sous-corpus 16, segment 2, page 19, ligne 18] (CFPQ)

(36) MY : ${ }^{2}$ nous-autres là là astheure euh: t'sais astheure où est-ce que je travaille c'est comme c'est la boss qui passe ça euh ${ }^{3} \mathrm{en}$ frais de représentation [sous-corpus 19 , segment 6, page 52, ligne 19] (CFPQ)

(37) $\mathrm{F}^{1} 1$ : et comment-ce que tu penses que $\left[\mathrm{P}_{4}\right]$ va réagir / ène fois que le deuxième bébé sera en train de nourrir là / quoi-ce que tu crois

$\mathrm{F}^{1} 3$ : ben vraiment asteure là / c'est manière de un petit jeu là chez nous / alle a des petits bébés partout partout là (corpus Petraș, 2016)

(38) Il a arrivé pis là astheur c'était le soir il a 'té à l'hôtel. (coll. Georges Arsenault, inf. Mme Jean L. Arsenault, 1975, Abram Village, Ile-du-Prince-Edouard, enreg. 922)

(39) - Ben non, non ça presse pas. Encore un autre! Envoyé un autre, paye pour un autre.

- Ah ! ben là astheur c'est fini, j'm'en vas là. (coll. Florida Arseneault Landry, inf. Virginie Bastarache, 1971, Saint-Thomas, Kent, Nouveau-Brunswick, bob. 1-2, enreg. 3).

Dans (37), (38) ou (39) ce sont les locutions discursives asteure là et là astheure qui se combinent avec d'autres marqueurs dans des associations libres.

\section{Asteure et à cette heure dans les français de France}

La démarche adoptée dans cette partie ne sera pas la même que dans la section antérieure. Car asteure ne connaît pas le même développement en France par rapport aux français nord-américains. Contrairement à ce qu'il en est dans ces derniers, asteure n'est pas enregistré dans les corpus de français contemporain de France. On ne le 
trouve pas dans un corpus comme ESLO (et ce quelle que soit la forme asteure, $\grave{a}$ $c^{\prime} t^{\prime} h e u r e^{9}$, astheure). Par contre, il est bien représenté pour la seconde moitié du $\mathrm{XX}^{\mathrm{e}}$ siècle dans la littérature régionale publiée dans Le Subiet par la Société d'ethnologie et de folklore du Centre-Ouest. Ainsi, on enregistre des attestations dans les numéros qui s'étalent de 1965 à 1999 (disponibles sur Gallica). Asteure est aussi attesté à partir de la fin du XIX ${ }^{e}$ siècle jusque dans les années 1940 dans Le Journal, quotidien littéraire et, à la même époque, dans d'autres journaux, dans les rubriques consacrées à la publication de récits régionaux ou à la publication de lettres de lecteurs (voir, par exemple, Le Matin, La Charente, La Croix de la Charente, Le Figaro sur Gallica) ou dans des revues consacrées à la publication de textes de la tradition populaire (voir, par exemple, La Mélusine, La tradition sur Gallica) .

Quant à l'expression à cette heure, elle est mentionnée dans TLFi, son emploi étant illustré par un exemple de V. Hugo. Le Nouveau Petit Robert (1996) la cite comme locution vieillie ou régionale pour la Belgique, proposant comme transcription phonétique [astœR] et comme sens «maintenant, présentement ». L'orthographe à cette heure ne rend pas compte de l'évolution s'étant déjà produite tant du point de vue formel que sémantique.

Une brève analyse des attestations et des fréquences respectives de asteure (astheure) et à cette heure dans la base Frantext, ainsi que des données offertes par les dictionnaires et les glossaires régionaux permet d'envisager l'émergence d'asteure et la dynamique de sa coexistence avec à cette heure.

Ast(h)eure coexiste avec à cette heure (FEW, $4: 468$ ), asteure-là « à ce moment-là ", à partir du moyen français (Palsgrave ${ }^{10}, 1530$, dans FEW) tant chez les différents auteurs que dans les parlers d'oïl (voir FEW, 4 : 468 ; DMF). Pourtant on ne le retrouve pas dans le corpus de moyen français de Frantext mais dans celui préclassique (Baïf, 1573, Montaigne, 1592, par exemple). D'ailleurs le DMF (1330-1500) qualifie asteure de « mot du XVI ${ }^{~ ", ~ d o n n a n t ~ d e s ~ e x e m p l e s ~ d e ~ B a i f ~ e t ~ d e ~ M o n t a i g n e . ~ S i ~ l ' o n ~ s e ~ r a p p o r t e ~ a u ~ m e ̂ m e ~}$ DMF (1330-1500), on voit bien que la construction (à) ceste heure présente déjà au XIV ${ }^{\mathrm{e}}$ et $\mathrm{XV}^{\mathrm{e}}$ siècles le sens "maintenant, en ce moment». Il faut mentionner que le Complément $d u$ Godefroy enregistre comme entrée asteure, tout en la caractérisant de " forme populaire et provinciale, à cette heure, à ce moment; se disait encore au dixseptième siècle ». La remarque concernant le caractère populaire de la forme est un élément important dans l'explication de la présence d'ast(h)eure au Canada.

Dans Frantext, asteure et astheure sont notamment attestés au XVI ${ }^{\mathrm{e}}$ et au XVII siècles : astheure n'est relevé que chez Jean Héroard, dans Histoire particulière de Louis XIII (1605-1610), paru en 1605, dans ses emplois déictique et anaphorique, et chez Nicolas de Peiresc, Lettres, tome 6, Lettres à sa famille, 1625. Chez ce dernier astheure coexiste avec asteure et avec à cette heure, dans les mêmes emplois. Astheure coexiste aussi avec maintenant dans une même phrase, avec une même signification. Pour ce qui est d'asteure, c'est chez Jean Antoine de Baïf (dans Le Brave, datant de 1573) qu'on retrouve le nombre le plus important d'occurrences (21, auxquelles s'ajoutent deux autres dans un autre écrit; sur ces 21 occurrences on enregistre 6 occurrences de tout asteure). Maintenant est aussi présent chez cet auteur.

Dans le tableau des fréquences à partir de Frantext, on remarquera qu'à part les cas déjà cités, asteure se retrouve chez Montaigne (5 occurrences) (voir un exemple dans 40 ). Chez ce dernier, asteure coexiste avec à cette heure. 
(40) C'est vrayement bien raison que cette experience commence à nous faillir. Qu'ils ne me reprochent point les maux qui me tiennent asteure à la gorge : d'avoir vescu sain quarante sept ans pour ma part, n'est ce pas assez (Montaigne, Michel de, Essais : t. 1 (livres 1 et 2), 1592, p. 764) Baguenault de Puchesse, asteure fait l'objet d'une correction (en note de l'éditeur) en à cette heure. Pour le $\mathrm{XVI}^{\mathrm{e}}$ siècle on l'enregistre aussi dans Recueil des lettres missives de Henri IV, tome IX (1567-1610), supplément publié par J. Guadet, 1876.

le $\mathrm{XIX}^{\mathrm{e}}$ siècle, asteure est recensé par différents glossaires régionaux ${ }^{11}$. On le retrouve aussi dans le journal Le Rigolo, dans une "Lettre saintong'haise » (no. 9/1883, sur Gallica). Il fait l'objet d'une réflexion métalinguistique dans Le Courrier de Vaugelas (1872-09-15), plus précisément dans le feuilleton Biographie des grammairiens pour la première moitié du XVII siècle, de Jean-Baptiste Duval : « Adverbes de temps. - En voici quelques-uns dont nous ne nous servons plus aujourd'hui : Asteure qu'employaient alors (1604) les poètes pour à ceste heure...»).

52 Asteure-cy fait figure de forme particulière dans La Prophécie du roy Charles VIII par Maitre Guilloche, publiée par le Marquis de La Grange, 1869, puisque la particule cy vient se joindre à la forme déjà contractée. siècle, notamment chez Raymond Queneau : dans Zazie dans le métro (4 occurrences, voir un exemple dans 41), Les fleurs bleues (3 occurrences), Les œuvres complètes de Sally Mara (2 occurrences).

(41) Les citoyens et citoyennes qui se trouvaient dans lcoin asteure se replièrent sur des positions moins esposées au tintouin (Zazie dans le métro, 1959, p. 138)

On le retrouve aussi chez Jean Vautrin (1 occurrence), dans Bloody Mary (1979) ou chez Jean-Patrick Manchette dans Journal : 1966-1974. discursif n'est pas absent, et ce dans les exemples provenant de la littérature régionale publiée dans Le Subiet (voir à cet égard 42).

(42) J'avis été cri des barbes aveuc Rosalie dans la forêt de Suza ; j'avis ma berouette et j'étis chargée aveuc le sa de Rosalie par d'sus les autes. J'étis obligée de faire des pauses. Asteure le foulait remette son sa su soune échine et je nous son disputés pour thieu pasque je me sens entêtée à vouloir tout zou rouler, et é m'a dit comme thieu [...] (Ine histouère dau pays de gueurnouilles, Le Subiet, 1981/01, p. 90, Gallica)

À partir de Frantext, l'analyse des différentes orthographes retenues par les écrivains rend compte d'une manière propre d'envisager la construction qui nous intéresse et partant ses significations. Ainsi, l'utilisation de l'expression à c't'heure par Raymond Queneau dans Le Chiendent (1933) ne correspond pas du tout aux significations identifiées pour asteure. Chez Queneau à c't'heure est une variante du syntagme prépositionnel à cette heure (non grammaticalisé), accompagné des particules là et ici (voir 43 et 44). 
(43) - Vous croyez qu'y aurait quéque chose qu'intéresserait un rupin comme le type blond?

- Sûr que non.

- Alors c'est pas drôle qu'i vienne à $c^{\prime} \boldsymbol{t}^{\prime}$ heure ici pour lui acheter quéque chose et qu'i veuille le réveiller pour ça ? (p. 89)

(44) Il s'assoit et frappe sur la table. Dans le fond, on grogne ; puis une femme assez immense s'amène.

«Je voudrais des frites. (Il prend l'offensive.)

- Des frites à c't'heure?»

Elle le regarde avec curiosité. Ce bourgeois, qui ça peut être ? (p. 25)

La transcription à c't'heure apparaît notamment chez des auteurs du $\mathrm{XX}^{\mathrm{e}}$ siècle. Un nombre considérable d'occurrences figure ainsi chez Roger Martin Du Gard, dans La Gonfle (1928), écrit particulier - farce paysanne - qui met en scène une parole oralisée. Contrairement à ce qu'il en est chez Raymond Queneau, chez Roger Martin du Gard à c't'heure présente un sens grammaticalisé, déictique, ou allant dans le sens discursif comme dans (45).

(45) Ah, ah, ah!...v'là vingt-sept années equ'ça dure, et puisqu'on va m'réparer ben conv'nablement ma Bique, à ç't'heure, j'ai bon espoir equ'ça n'est point'core près ed'finir... l'âge, i'n'détourne point ed'la galantise autant qu'on a coutume ed'dire... non!

Et nous n'somm'point, la Bique et moi, coumme les saints et sainte'ed'l'almanaque, qui sont toute l'année couchés côte à côte sans (p. 1225)

Le sens discursif émergent se manifeste aussi chez George Chepfer, dans Saynètes, paysanneries 2 (1945), Le tonton est collectionneur (p. 245) (voir 44).

(46) On écouterait les femmes qu'on ne ferait jamais rien de bien.

- Dis-voir que ce n'est pas une manie de garder toutes les vieilleries-là qu'on ne ramasserait pas sur le tas, depuis que t'as trouvé trois quatre vieilles pièces de monnaie qui datent de Mathusalem en rabourant sur la côte.

A c't'heure, monsieur Bossatte, il ne pense pus qu'à chercher des trésors cachés et il passe tout son temps à mettre nos champs sens dessus-dessous, et je te fouille et je te refouille, c'est toi qui te fouilleras à la fin. [...]

Pour le XIX ${ }^{e}$ siècle, à c't'heure est attesté chez Honoré de Balzac, dans Le Père Goriot (1843), chez Guy de Maupassant, dans Une vie (1883), Contes et nouvelles (1885) et Contes du jour et de la nuit (1885). Au XVIII ${ }^{\text {e }}$ siècle, on retrouve à c't'heure chez Dorvigny, dans Les battus paient l'amende (1779) ou chez Nicolas Rétif de la Bretonne, dans La paysanne pervertie, ou Les dangers de la ville (1784).

Pour ce qui est la présence de à cette heure dans Frantext, comme locution, avec une signification déictique, l'expression est d'un emploi constant à partir du XVI siècle (par exemple, chez Montaigne, en alternance avec asteure) jusqu'au XX $\mathrm{X}^{\mathrm{e}}$ siècle.

\section{Conclusion : changement linguistique et parcours de grammaticalisation}

61 Les quelques observations concernant le fonctionnement et l'évolution de l'expression asteure tant dans les variétés de français du Canada qu'en français de France, du point de vue synchronique et partiellement diachronique, nous permettent d'envisager, dans un premier temps, l'émergence de l'adverbe ast(h)eure à partir de l'expression à cette heure. Il s'agit d'une grammaticalisation de longue date (dès le Moyen Français), dont rendent compte des phénomènes tels la coalescence, l'érosion phonétique, la 
paradigmatisation. S'y ajoutent le changement sémantique d'un sens transparent (calculable à partir du sens des éléments composants de l'association libre) vers un sens plus abstrait (déictique), ainsi que le déplacement de position en tête de phrase (voir 47). Asteure en tête de phrase a une valeur ambiguë : c'est probablement de là que la valeur de marqueur discursif émerge. Pour ce qui est de l'évolution sémantique, on remarque la continuité entre la valeur déictique conférée par le déterminant démonstratif dans la construction à cette heure et la valeur déictique de l'adverbe asteure.

(47) Il sçait bien qu'il n'a pas tenu à moy et je crois certainement qu'il aung peu negligé ceste affaire et que s'il y eus thazardé la despanse du voyage d'ung homme pour aller traicter avec ceux qui luy pouvoyent mettre en main la pièce qu'on luy demandoit, il en seroit venu à bout. Asteure je me doubte qu'il ne soit ung peu trop tard pour s'en adviser. Toutesfois s'il n'y plaignoit poinct ung peu de despance, au hazard, possible y trouveroit il encores quelque entree, maison ne peult pas aisement traicter de cez choses là de si loing par simples lettres sans entremise d'hommes. (Nicolas de Peiresc, Lettres : t. 5 : 1610-1637 : Lettres à Guillemin, 1637, p. 40)

Loin de représenter une particularité des variétés de français nord-américain, asteure, avec sa signification déictique est un héritage de France, où l'adverbe se maintient d'ailleurs tout le long du $\mathrm{XX}^{\mathrm{e}}$ siècle dans la littérature régionale et est récupéré par certains auteurs.

Deuxièmement, on remarque l'évolution d'asteure vers l'emploi de marqueur discursif, notamment dans les français nord-américains. Ce n'est pas un cas singulier. Tout au contraire, asteure s'inscrit, avec maintenant ou or, dans la catégorie des adverbes de temps qui en viennent à fonctionner aussi comme marqueurs discursifs, anaphoriques, de relation. À son tour, le marqueur discursif asteure se combine avec d'autres marqueurs, contribuant à l'émergence d'associations liées ou libres. L'évolution vers une valeur discursive parallèlement avec la contraction et la coalescence indique une grammaticalisation avancée. La valeur discursive est d'ailleurs incompatible avec la construction à cette heure.

Lorsqu'on s'interroge sur l'évolution d'un emploi proprement adverbial à l'emploi comme marqueur discursif, l'exemple (48) tiré du conte de tradition orale déjà mentionné, offre un cas intéressant, à rapprocher de l'emploi comme ponctuant. Apparemment, astheur vient clôturer un syntagme, qui est probablement repris et étoffé après coup (aux noces...du roi). Cette liberté de position peut être considérée comme une étape dans le processus de grammaticalisation qui conduit à l'émergence du marqueur discursif.

(48) Quand qu'i avont arrivé là, p'tit Jack a arrivé aux noces, astheur, du roi, i a monté pour prendre son joual par la bride pour le... (La Bête à sept têtes)

Pour ce qui est du succès d'asteure dans les français nord-américains, sa structure transparente pourrait avoir joué en sa faveur. Il faut invoquer aussi la nature du parler transporté au Canada. Il s'agit d'un français empreint d'éléments dialectaux et populaires. Or, asteure est attesté dans les parlers d'oïl et en français populaire de France, ce qui vient confirmer le fait qu'il s'agit, au Canada, d'un héritage de France, où l'adverbe reste cantonné à un emploi régional. Pourtant, asteure connaît dans les français nord-américains des développements propres, qui montrent bien la dynamique interne de ces variétés. Elles se singularisent et s'autonomisent par rapport 
au français de France, du fait d'évolutions sous l'influence de facteurs socio-historiques propres (rapport particulier à la norme, caractère populaire des éléments transportés).

\section{BIBLIOGRAPHIE}

ARRIGHI, L., 2005, Étude morphosyntaxique du français parlé en Acadie - Une approche de la variation et du changement linguistique en français, thèse de doctorat, Université d'Avignon. BERTHONNEAU, A.-M., KLEIBER, G., 1993, « Pour une nouvelle approche de l'imparfait : l'imparfait, un temps anaphorique méronomique », Langages, 112, p. 55-73.

BERTIN, A., 2001, « Maintenant : un cas de grammaticalisation? », Langue française, 130, p. 42-64. BRASSEUR, P., 2001, Dictionnaire des régionalismes du français de Terre-Neuve, Tübingen, Max Niemeyer Verlag.

CHEVALIER, G., HUDSON, C., 2005, « Deux cousins en français québécois et en chiac de Moncton : right et full », dans P. Brasseur et A. Falkert (éds.), Français d'Amérique : approches morphosyntaxiques, Paris, L'Harmattan, p. 289-302.

CLAPIN, S., 1894, Dictionnaire canadien-français, Montréal, C. Beauchemin.

CORMIER, Y., 1999, Dictionnaire du français acadien, Montréal, Éditions Fides.

DIONNE, N.-E., 1974, Le parler populaire des Canadiens français, Québec, Les Presses de l'Université Laval (reproduction en fac-similé de l'édition de 1909, Québec, LaFlamme \& Proulx, Imprimeurs).

DUNN, O., 1880, Glossaire franco-canadien et vocabulaire de locutions vicieuses usitées au Canada, Québec, Imprimerie A. Côté et Cie.

DMF = Dictionnaire du Moyen Français (1330-1500) (2015). http://www.atilf.fr/dmf/

DOSTIE, G., 2004a, Pragmaticalisation et marqueurs discursifs. Analyse sémantique et traitement lexicographique, Bruxelles, De Boeck/Duculot.

DOSTIE, G., 2004b, « Considération sur la forme et le sens. Pis en français québécois. Une simple variante de puis? Un simple remplaçant de et? », French Language Studies, 14. p. 113-128.

DOSTIE, G., 2007, « La réduplication pragmatique des marqueurs discursifs. De là à là là », Langue française, 154/2, p. 45-60.

DOSTIE, G., 2013, « Les associations de marqueurs discursifs. De la cooccurrence libre à la collocation », Linguistik online, 62/5, p. 15-45.

DOSTIE, G., 2015, « Réflexions sur la (quasi-)synonymie et la variation diaphasique. L'exemple de et/pis en français québécois familier », dans G. Dostie et P. Hadermann (éds.), La dia-variation en français actuel. Étude sur corpus, approches croisées et ouvrages de référence, Berne, Peter Lang, p. 147-177.

FALKERT, A., 2006, « La mutation achevée du connecteur ça fait que dans le français acadien des ̂̂les-de-la-Madeleine », Revue canadienne de linguistique appliquée, 9 (2), p. 39-53. 
FEW = Von Wartburg W. (1968-1989), Französisches Etymologisches Wörterbuch. Eine Darstellung des galloromanischen Sprachschatzes, Basel, Zbinden.

Glossaire du parler français au Canada (1968) (préparé par la Société du parler français au Canada), Québec, Les Presses de l'Université Laval (reproduction en fac-similé de l'édition de 1930, Québec, L'Action sociale).

GODEFROY, F., 1895, Dictionnaire de l'ancienne langue française et de tous ses dialectes du IX ${ }^{\mathrm{e}}$ au $\mathrm{XV}^{\mathrm{e}}$ siècle. Complément, Paris, F. Vieweg, libraire-éditeur. Tome huitième. Consulté à http:// micmap.org/dicfro/search/complement-godefroy/

KLEIBER, G., 2003, « Entre les deux mon cœur balance ou L'imparfait entre aspect et anaphore », Langue française, 138 , p. 8-19.

LAURENDEAU, P., 1983, « De l'adverbe de temps au joncteur logique : asteur et d'abord en québécois », Revue de l'Association québécoise de linguistique, 3/2, p. 143-158.

NEUMANN-HOLZSCHUH, I., MITKO, J., 2018, Grammaire comparée des français d'Acadie et de Louisiane. Avec un aperçu sur Terre-Neuve, Berlin/Boston, De Gruyter.

Le Nouveau Petit Robert (1996) (texte remanié et amplifié sous la direction de Josette Rey-Debove et Alain Rey), Paris, Dictionnaires Le Robert.

PETRAș, C., 2016, Contact de langues et changement linguistique en français acadien de la Nouvelle-Ecosse. Les marqueurs discursifs, Paris, L'Harmattan.

SÉGUIN, A., 2011, « Désormais, dorénavant et asteure : variation aspectuo-temporelle », Communication, lettres et sciences du langage, 5/1, p. 137-147.

TLFi : Trésor de la langue française informatisé, conception et réalisation informatiques Jacques Dendien, http://atilf.atilf.fr

WALTEREIT, R., 2007, « À propos de la genèse diachronique des combinaisons de marqueurs. L'exemple de bon ben et enfin bref », Langue française, 154/2, p. 94-109.

WISSNER, I., 2017, « Le marqueur là en français actuel dans l'espace francophone : quelle description lexicographique pour quel(s) usage(s)?», Revue de Sémantique et Pragmatique, 41-42, p. 79-97.

\section{Corpus}

Contes de tradition orale, transcriptions. Archives d'ethnologie et de folklore, Centre d'Études Acadiennes Anselme-Chiasson, Université de Moncton.

Corpus Arrighi (2005) = Arrighi L. (2005). Étude morphosyntaxique du français parlé en Acadie : une approche de la variation et du changement linguistique en français. Thèse de doctorat. Université d'Avignon. Tome II : corpus (1), tome III : corpus (2)

Corpus ESLO = Enquêtes Sociolinguistiques à Orléans. http://eslo.huma-num.fr/index.php Corpus Falkert (2010) = Falkert A. (2010). Le français acadien des îles-de-la-Madeleine . Étude de la variation phonétique. Paris : L'Harmattan. Corpus (CD-ROM)

CFPQ = Dostie G. et al. (2006-2015). Corpus de français parlé au Québec. Université de Sherbrooke, Centre d'analyse et de traitement informatique du français québécois renommé en 2016 Centre de recherche interuniversitaire sur le français en usage au Québec. https:// applis.flsh.usherbrooke.ca/cfpq/ 
Corpus Petraş (2016) = Petraş C. (2016). Contact de langues et changement linguistique en français acadien de la Nouvelle-Écosse. Les marqueurs discursifs. Paris : L'Harmattan. Corpus (CDROM).

Corpus Wiesmath (2006) = Wiesmath R. (2006). Le français acadien. Analyse syntaxique d'un corpus oral recueilli au Nouveau-Brunswick/Canada. Paris : L'Harmattan. Corpus (CD-ROM).

Frantext. https://www.frantext.fr.

Gallica. gallica.bnf.fr.

$\mathrm{TLFQ}=$ Trésor de la langue française au Québec. http://www.tlfq.ulaval.ca/fichier/

\section{NOTES}

1. Cet article a été réalisé dans le cadre d'un projet de recherche financé par le Ministère de la Recherche, de l'Innovation et de la Numérisation, CNCS/CCCDI - UEFISCDI, dans le cadre du PNCDI III, numéro du projet PN-III-P4-ID-PCE-2020-1505.

2. Nous retenons l'orthographe asteure (avec les variations astheure et astheur) qui rend compte d'une grammaticalisation très avancée à partir de l'expression à cette heure.

3. Ainsi, dans l'ensemble des transcriptions des contes de tradition orale acadiens réunies au Centre d'Études Acadiennes Anselme-Chiasson de l'Université de Moncton on compte une cinquantaine d'occurrences de maintenant.

4. Se servant des instruments d'une approche des opérateurs dans les langues naturelles, Laurendeau (1983) analyse le "glissement sémantique » qui sous-tend le passage du rôle d'«adverbe de temps» à celui de «joncteur logique interpropositionnel » dans le cas d'adverbes comme asteur, d'abord, finalement, ensuite. Selon cet auteur, " il y a dans la sémantèse initiale de chaque adverbe de temps des éléments de contenu sémantique qui déterminent déjà ce que sera leur rôle de joncteur logique » (p. 148). Ainsi, on peut facilement interpréter l'ordre chronologique comme un rapport logique causal. D'autres valeurs sont identifiées : asteur peut fonctionner comme «marqueur d'incompatibilité » entre deux énoncés dans ce que Laurendeau appelle « refus d'implication » (pp. 151-152) ; l'adverbe peut aussi apparaître dans un emploi métalinguistique de «marqueur d'inconséquence, de non pertinence » (p. 152), dérivé de l'emploi antérieur.

5. «[...] on peut conclure à la polysémie de maintenant, le lien d'un sens à l'autre se rattachant à une structure métonymique : de $\mathrm{T}_{0}$ à $\mathrm{T}_{\mathrm{i}}$ par élargissement de l'instant en moment (II-1.), du temporel au renforcement d'un acte de parole (affirmation, ordre, interrogation) ou à l'opposition par l'intermédiaire d'un lien de conséquence ou d'opposition entre ce qui est posé en $\mathrm{T}_{0}$ et le verbe de l'énoncé (II-2.) » (pp. 59-60).

6. Il s'agit du conte La bête à sept-têtes de la Collection Georges Arsenault, recueilli à Mont-Carmel, Ile du Prince-Edouard, le 30 juin 1975, inf.: Augustin Gallant (69), Bob. 59, enreg. 933). Nous gardons l'orthographe proposée par les transcripteurs.

7. Nous reviendrons dans 1.3 sur les associations auxquelles donne lieu asteure.

8. On enregistre droit' icitte dans le corpus Falkert (2010).

9. À cette heure ne figure que dans à cette heure-là.

10. Lesclaircissement de la Langue Françoyse : asteure = à cette heure, maintenant.

11. Voir, par exemple, le Glossaire étymologique et comparatif du patois picard, ancien et moderne..., par l'abbé Jules Corblet, 1851 ; le Glossaire de la Vallée d'Yères, pour servir à 
l'intelligence du dialecte haut-normand..., par A. Delboulle, 1876 ; Remarques sur quelques expressions usitées en Normandie: leur emploi par certains auteurs, leur origine, leur étymologie, par Gustave Le Vavasseur, 1878 (a c'theure, asteure) ; le Dictionnaire du langage populaire verduno-chalonnais (Saone-et-Loire), par F. Fertiault, 1890.

\section{RÉSUMÉS}

L'article propose une mise en parallèle du fonctionnement de l'expression asteure dans les français nord-américains et de son évolution en français de France. L'analyse aboutit à la conclusion de l'émergence de l'adverbe ast(h)eure à partir de l'expression à cette heure, à la suite d'une grammaticalisation de longue date. Dans un deuxième temps, on remarque l'évolution de l'adverbe de constituant asteure vers l'emploi comme marqueur discursif notamment dans les français nord-américains, ce qui rend compte d'une grammaticalisation avancée. Asteure s'inscrit ainsi dans la catégorie des adverbes de temps qui fonctionnent aussi comme marqueurs discursifs.

The article offers a comparison between the functioning of the expression asteure in North American French and its evolution in French from France. The analysis leads to the conclusion of the emergence of the adverb ast(h)eure from the expression à cette heure, as a result of longstanding grammaticalization. Secondly, we notice the evolution of the temporal adverb asteure towards its use as a discursive marker, particularly in North American French, which reflects an advanced grammaticalization. Asteure thus falls into the category of temporal adverbs which also function as discursive markers.

\section{INDEX}

Mots-clés : asteure, marqueur discursif, variétés de français nord-américain, grammaticalisation, locutions discursives

Keywords : asteure, discourse marker, varieties of North American French, grammaticalization, discursive phrases

\section{AUTEUR}

\section{CRISTINA PETRAS}

Université Alexandru Ioan Cuza Iași 